\title{
Investigation of Lapses of Consciousness using a Tracking Task: Preliminary Results
}

\author{
Malik T. R. Peiris ${ }^{1,2}$, Richard D. Jones ${ }^{1,2}$, Grant J. Carroll ${ }^{3}$, Philip J. Bones ${ }^{1}$ \\ ${ }^{1}$ Department of Electrical and Computer Engineering, University of Canterbury, Christchurch, New Zealand \\ ${ }^{2}$ Department of Medical Physics \& Bioengineering, Christchurch Hospital, Christchurch, New Zealand \\ ${ }^{3}$ Department of Neurology, Christchurch Hospital, Christchurch, New Zealand
}

\begin{abstract}
In many high-risk occupations, it is critical that a person remains alert at all times. There is much to be gained by being able to monitor a person on-line and detect lapses of consciousness (LoC) so that remedial action can be taken (e.g., a rest break) to ensure that safety is maintained. In this study, 15 normal subjects were observed on two sessions while they performed a continuous tracking task for a period of 1 hour. EEG, eye movements, tracking performance data and a video of the subject were recorded during the session. This paper presents some preliminary results on the phenomenon of lapsing. Only 4 of the 15 subjects did not have a LoC at some stage. Seven subjects had LoCs more than 45 times and 4 more than 100 times during the 2 hours. The mean rate of lapsing over all subjects was $29.1 \mathrm{LoC} / \mathrm{h}$. In contrast, lapses in performance were caused by both lapses of consciousness $(30.1 \%)$ and attention $(69.9 \%)$. There was no correlation found between age of subject and number of lapses of consciousness.
\end{abstract} tracking

Keywords-Alertness, lapses of consciousness, visuomotor

\section{INTRODUCTION}

People in high-risk jobs such as truck drivers, locomotive drivers, pilots, health professionals and others providing 24-h services must remain alert at all times. Diminished alertness levels in these occupations can lead to disastrous consequences such as multiple fatalities. Cases of tired drivers falling asleep while driving are not uncommon. Furthermore, there is considerable anecdotal evidence, often from personal experience, of brief lapses of consciousness or 'micro-sleeps' during everyday tasks ranging from driving a vehicle to attending a talk or lecture. Thus, a system able to continuously monitor a person on-line and detect lapses of consciousness would be of considerable value in helping to prevent serious accidents.

In contrast to alertness/drowsiness estimation [1-6] and sleep detection [7-9], few techniques [10] appear able to identify brief lapses of consciousness (cf. lapses in performance).

It has been shown that increasing the duration of the time-on-task [2], using a continuous tracking task (CTT), as well as providing conditions conducive to sleeping, can increase the probability of a subject lapsing. Subjects sitting

This research was financially supported by the Christchurch Neurotechnology Research Programme and the University of Canterbury, Christchurch, New Zealand. in a comfortable chair in a quiet, dimly-lit, warm room and asked to refrain from making unnecessary movements, tend to lose vigilance and have intermittent drop-offs in task performance $[5,11,12]$.

In the current study, we are exploring the phenomenon of lapsing using full-head EEG, eye movements, and video recording on a subject carrying out a CTT. A key aim of the study was to obtain physiological measurements from subjects when they have lapses. It was hypothesized that (a) some subjects undertaking the CTT for an extended period of time and in an environment conducive to sleeping would have occasional lapses of consciousness and (b) there may be physiological cues during lapses in the EEG or eye movements. This paper focuses on performance lapses definitively caused by lapses of consciousness. This was achieved by employing an independent measure (video observation of facial features and eye movements) to eliminate performance lapses caused by lapses of attention.

This paper presents preliminary data on the presence and characteristics of lapses in performance, lapses of attention, and lapses of consciousness during an extended CTT.

\section{Methodology}

\section{A. Subjects}

Fifteen normal healthy male volunteers aged 18-36 years $($ mean $=26.5)$ who did not have a current or previous neurological or sleep disorder were chosen for the study. All subjects had visual acuities of $6 / 9(=20 / 30)$ or better in each eye.

Subjects were asked to refrain from consumption of stimulants/depressants, such as caffeine, nicotine, alcohol, illicit drugs, and medications, within 4 hours prior to the two study sessions.

All experimental sessions were held following lunch during the period 12.30 p.m. to 5.00 p.m.

Ethical approval for the study was obtained from the Canterbury Ethics Committee.

\section{B. Apparatus}

The tracking system consisted of a Pentium PC with two 17" colour monitors, a Matrox G550 dual-head graphics card, and an Advantech PCL 1710 data acquisition board. 
The tracking task was generated by the program SMTests ${ }^{\mathrm{TM}}$ [13] and a steering wheel (395 $\mathrm{mm}$ diameter) was used to measure the subject's tracking movements.

A random tracking task with an 8-s preview [14] was chosen as the optimal CTT for this study. This task measures a subject's ability to keep an arrow point on a pseudo-random target waveform (bandwidth $0.156 \mathrm{~Hz}$, period $64 \mathrm{~s}$ ) moving vertically down the screen. The task requires smooth movements over a 175-degree range of the steering wheel. The subject's response was sampled at 64 $\mathrm{Hz}$ and stored on disk.

Changes in facial expression, head position, etc., were observed on video to provide an independent, albeit subjective, measure of fatigue, lapses of consciousness, and sleep [15].

EEG and EOG were also recorded during the study but are not discussed further in this paper.

\section{Procedure}

The requirements of the tracking task were explained to the subject and they were asked to stay alert and perform to the best of their ability and, aside from eye blinks, to keep their eyes open as much as possible during the task. Their objective was to track the random target on the screen using the steering wheel for a period of one hour. The subjects sat in a comfortable chair during the session and the room temperature was maintained between $22-25^{\circ} \mathrm{C}$.

At the end of a session the subject was asked how drowsy they felt they had become during the tracking and whether they felt they had lapsed at any point during the session.

Each subject attended two sessions held on different days (separated by a mean of 17 days, range 7-50).

\section{Types of Lapses}

For conceptual and analytical purposes we have defined three types of lapses: (1) Lapse in performance (LiP) - a 'prolonged period' ( $>1.0 \mathrm{~s})$ during which the participant is unequivocally not responding appropriately to the changing target in the continuous tracking task; (2) Lapse of consciousness (LoC) - a temporary ( $>1.0 \mathrm{~s})$ complete loss of responsiveness as indicated by a LiP, occurring concurrently with an apparent loss of consciousness as determined independently by video observation; (3) Lapse of attention (LoA) - a loss of responsiveness which cannot be attributed to a LoC during which the subject appears conscious according to independent video observation.

An important distinction between a LiP and a LoC is that a LiP can be measured using a task such as the psychomotor vigilance task (PVT) [16] or the CTT but a LoC cannot be unequivocally determined through such tasks alone. For example, a subject can exhibit a LiP despite being fully alert due to a LoA caused by an overt distraction or simply by thinking about something other than the task. That is, a substantial LiP can be due to either a LoC or LoA.

\section{E. Analysis}

A fuzzy-rule based system was used to calculate a measure of the subject's tracking performance. A section of tracking data was classified as a LiP if (1) there was a sudden increase in the absolute error $\left(\mathrm{E}_{\mathrm{TU}}\right)$ between the target $(\mathrm{T})$ and the user response $(\mathrm{U})$ for a period $>1 \mathrm{~s}$ (time threshold $t_{L}$ ) and with no evidence of correction and (2) if $U$ was static for a period greater than $t_{L}$ while $T$ was changing. These rules were used to generate a performance index which can vary between 0 (poor) and 1 (excellent).

LiPs identified by the fuzzy system were compared with independently rated video recordings of the CTT sessions. The video was classified into one of 7 video-based levels of alertness: alert $\left(A_{V}\right)$, distracted $\left(\operatorname{Dis}_{V}\right)$, forced eye closure while alert $\left(\mathrm{FEC}_{\mathrm{V}}\right)$, light drowsy $\left(\mathrm{LD}_{\mathrm{V}}\right)$, deep drowsy $\left(\mathrm{DD}_{\mathrm{V}}\right)$, lapse of consciousness $\left(\mathrm{L}_{\mathrm{V}}\right)$, and sleep $\left(\mathrm{S}_{\mathrm{V}}\right)$. These levels are, of course, subjective and based on observing the eye movements and facial expressions of the subject during the tracking task. Based on video alone, choosing between $\mathrm{LD}_{\mathrm{V}}$ and $\mathrm{DD}_{\mathrm{V}}$ was found to be particularly difficult. Similarly, the distinction between $\mathrm{L}_{\mathrm{V}}$ and $\mathrm{S}_{\mathrm{V}}$ was arbitrary since it is mainly based upon the duration of head-drops and how a subject 'wakes' from a lapse or sleep.

Finally, the presence of a LoC was determined by the coincidence of a LiP and $\mathrm{L}_{\mathrm{V}}$ or a $\mathrm{LiP}$ and $\mathrm{S}_{\mathrm{V}}$.

\section{RESUlts}

\section{A. Lapse profile}

Fig. 1 shows the combined-session LiP, $\mathrm{L}_{\mathrm{V}}$, and LoC totals for each of the 15 subjects. There was an overall total of $2906 \mathrm{LiPs}, 30.1 \%$ of which could be attributed to LoC and $69.9 \%$ to LoA. Only 4 of the 15 subjects did not have a LoC at some stage. Seven subjects had LoCs more than 45 times and 4 more than 100 times during the 2 hours studied. The mean rate of lapsing over all subjects was $29.1 \mathrm{LoC} / \mathrm{h}$ (range 0-147). The mean duration of a LoC was $4.0 \mathrm{~s}$ (range 1.0-77.0 s).

\section{B. Correlation between Lapsing and Age of Subject}

Fig. 2 shows the number of LoCs versus the age of the subject. There is no correlation between LoC and age $(r=$ $0.048, p=0.87$ ), indicating that propensity for lapsing is unrelated to age, at least over the range of age studied.

\section{Differences between Sessions}

We hypothesised that subjects would tend to have more LoCs on the second session than the first, due to factors such as boredom and familiarity with the surroundings. 


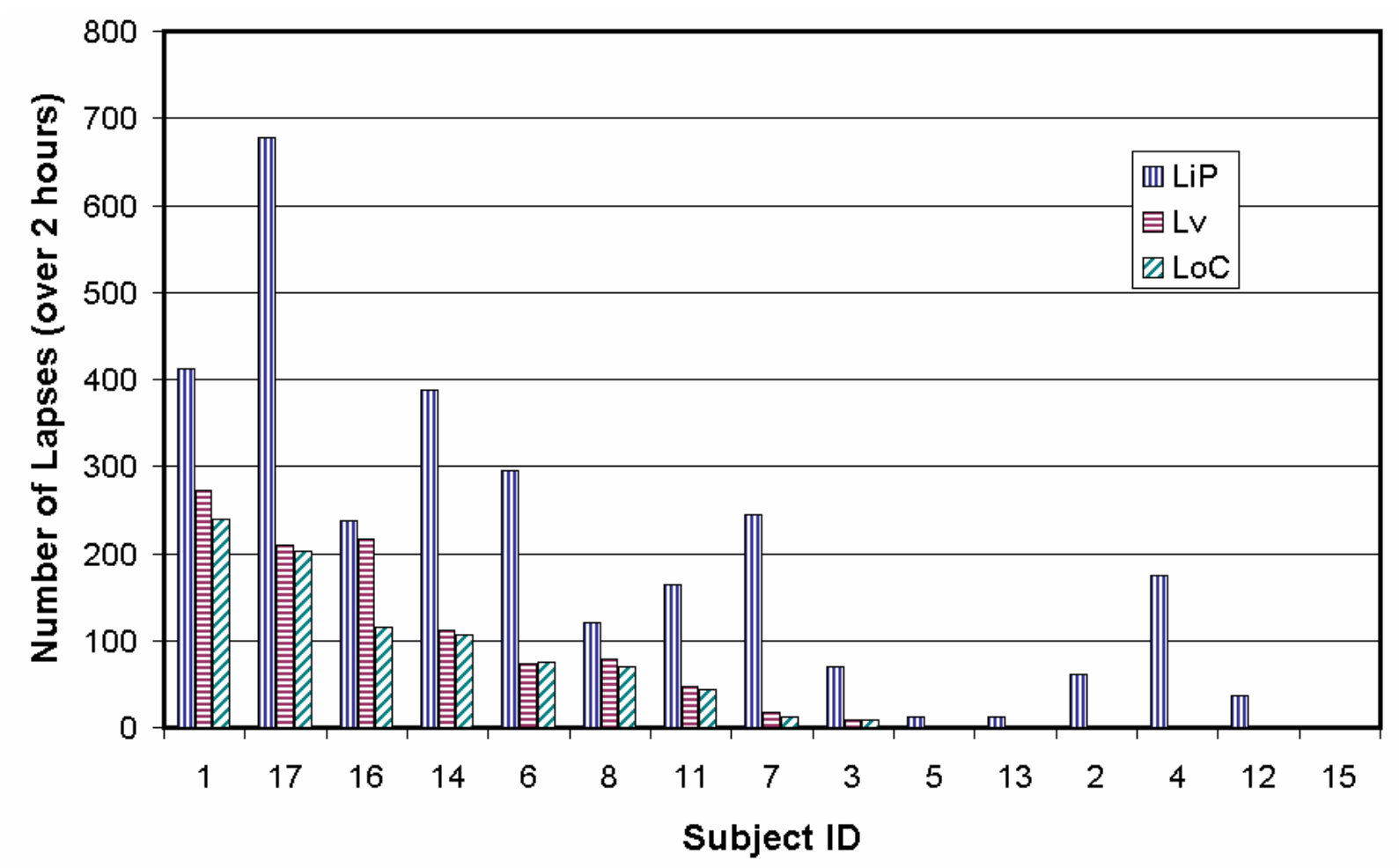

Fig. 1. Number of lapses in performance (LiP), video-based lapses of consciousness $\left(\mathrm{L}_{\mathrm{V}}\right)$, and video-confirmed lapses in performance due to lapses of consciousness (LoC) for the 15 subjects (both sessions)

However, there was no difference in the number of LoCs between sessions 1 and 2 (25.5 vs. 32.9, $p=0.53)$.

Although there was no overall difference in the number of LoCs between the first and the second sessions, there was substantial variability between the best and worst session of each subject (18.0 vs. 40.3, $p=0.0033)$.

\section{Self-awareness of Lapses}

After completing the tracking, subjects were asked whether they were aware of any lapses of consciousness during the session. All subjects who had had one or more LoC mentioned that they were aware of having lapsed at one

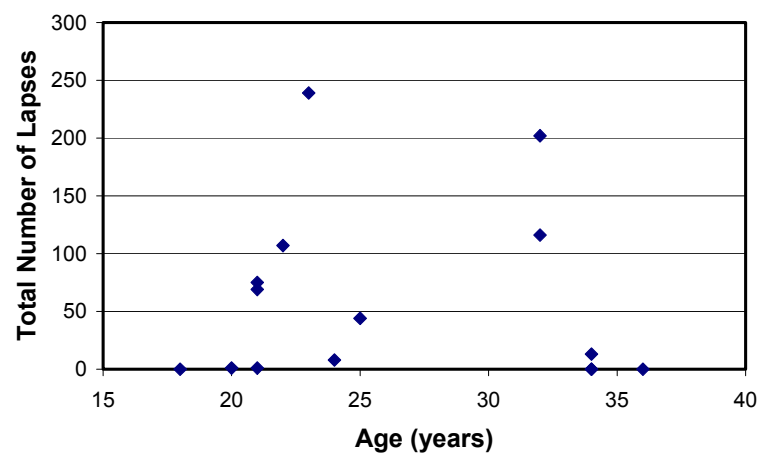

Fig. 2. Lapses in consciousness over both sessions versus age of subject. or more points during the session. Conversely, 5 subjects considered that they had lapsed in 8 of 10 sessions in which no LoCs were evident. There were, however, LoAs in all 8 of these sessions.

\section{DiscUSSION}

These results show that most subjects (11 out of 15$)$ had a LoC one or more times during the two 1-hour tracking sessions, with 7 subjects lapsing at $22 \mathrm{LoC} / \mathrm{h}$ or more. This was not at all expected from a group of healthy, non-sleepdeprived individuals performing a tracking task during normal work hours.

LiPs were found to be due to both LoC (30\%) and LoA $(70 \%)$. It is not a surprising that the majority of LiPs can be attributed to a LoA due to the monotonous nature of the CTT.

There was considerable variability in the extent of lapsing between subjects. However, this does not seem to be related to age, although the age range of our subjects was intentionally relatively narrow (18-36 years).

Although we expected the subjects to lapse more during the second session, this proved to not be the case. Although subjects presumably found the second tracking session more boring than the first, this did not seem to affect their performance, at least in terms of lapses of consciousness. 
Conversely, subjects varied considerably in the number of LoCs they had during the two sessions.

All subjects who had one or more LoCs were aware that they lapsed during the session. This agrees with anecdotal evidence of drivers being aware that they have briefly 'nodded off' while driving at night. However, 5 subjects thought they had a LoC when no LoCs were evident. Therefore, subjects tend to over-emphasize LoC events. This indicates that (a) subjects had a tendency to overestimate lapses of consciousness or (b) subjects included LiPs during deep drowsiness or (c) there were indeed additional LoCs but these were not definitively apparent on the video recordings.

\section{CONCLUSION}

Despite some apprehensiveness about whether subjects would lapse at all during the 1-h tracking task, this study indicates that lapses of consciousness can occur in young healthy adults to a much greater extent than previously recognized. This has major implications for occupations that require sustained alertness over long periods of time. From a safety point of view, it would clearly be very desirable to counter such lapses by being able to provide a means of early detection of lapses.

\section{ACKNOWLEDGMENT}

The authors wish to thank Cathy Lai for customizing the tracking software.

\section{REFERENCES}

[1] R. Conradt, U. Brandenburg, T. Penzel, J. Hasan, A. Värri, and J. H. Peter, "Vigilance transitions in reaction time test: a method of describing the state of alertness more objectively," Clin. Neurophysiol., vol. 110, pp. 1499-1509, 1999.

[2] S. M. Doran, H. P. Van Dongen, and D. F. Dinges, "Sustained attention performance during sleep deprivation: evidence of state instability," Arch. Ital. Biol., vol. 139, pp. 253-67, 2001.

[3] T. Jung, S. Makeig, M. Stensmo, and T. Sejnowski, "Estimating Alertness from the EEG Power Spectrum," IEEE Trans. Biomed. Eng., vol. 44, pp. 60-69, 1997.

[4] S. Makeig and M. Inlow, "Lapses in alertness: coherence of fluctuations in performance and EEG spectrum," Electroencephalogr. Clin. Neurophysiol., vol. 86, pp. 23-35, 1993.

[5] S. Makeig and T.-P. Jung, "Tonic, phasic, and transient EEG correlates of auditory awareness in drowsiness," Brain Res. Cogn. Brain Res., vol. 4, pp. 15-25, 1996.

[6] A. Vuckovic, V. Radivojevic, A. C. Chen, and D. Popovic, "Automatic recognition of alertness and drowsiness from EEG by an artificial neural network," Med. Eng. Phys., vol. 24, pp. 349-60, 2002.

[7] L. De Gennaro, M. Ferrara, F. Ferlazzo, and M. Bertini, "Slow eye movements and EEG power spectra during wake-sleep transition," Clin. Neurophysiol., vol. 111, pp. 2107-2115, 2000.
[8] L. Torsvall and T. Akerstedt, "Sleepiness on the job: continuously measured EEG changes in train drivers," Electroencephalogr. Clin. Neurophysiol., vol. 66, pp. 502-511, 1987.

[9] L. De Gennaro, M. Ferrara, and M. Bertini, "The boundary between wakefulness and sleep: quantitative electroencephalographic changes during the sleep onset period," Neuroscience, vol. 107, pp. 1-11, 2001.

[10] M. C. Moore-Ede, U. E. Trutschel, R. Guttkuhn, and A. Heitmann, (2003). U.S. Patent No. 6,511,424 B1. U.S. Patent and Trademark Office.

[11] V. Valley and R. Broughton, "The physiological (EEG) nature of drowsiness and its relation to performance deficits in narcoleptics," Electroencephalogr. Clin. Neurophysiol., vol. 55, pp. 243-251, 1983.

[12] K. F. Van Orden, T.-P. Jung, and S. Makeig, "Combined eye activity measures accurately estimate changes in sustained visual task performance," Biol. Psychol., vol. 52, pp. 221 - 240, 2000.

[13] R. D. Jones, N. B. Sharman, R. W. Watson, and S. R. Muir, "A PCbased battery of tests for quantitative assessment of upper-limb sensory-motor function in brain disorders," in Proc. 15th Int. Conf. IEEE Eng. Med. Biol. Soc., San Diego, 1993. 1414-1415.

[14] R. D. Jones, "Measurement of sensory-motor control performance capacities," in The Biomedical Engineering Handbook, vol. 2, J. D. Bronzino, Ed., 2 ed. Boca Raton, Florida: CRC Press, 2000, pp. 149:125 .

[15] S. K. L. Lal and A. Craig, "A critical review of the psychophysiology of driver fatigue," Biol. Psychol., vol. 55, pp. 173 - 194, 2001.

[16] D. F. Dinges, M. M. Mallis, G. Maislin, and J. W. Powell, "Evaluation of techniques for ocular measurement as an index of fatigue and as the basis for alertness management," National Highway Traffic Safety Administration, Washington, DC. Final report DOT HS 808 762. 1998. 